\title{
Helical jets in blazars
}

\section{Interpretation of the multifrequency long-term variability of $\mathrm{AO} 0235+16$}

\author{
L. Ostorero ${ }^{1}$, M. Villata $^{2}$, and C. M. Raiteri ${ }^{2}$ \\ ${ }^{1}$ Landessternwarte Heidelberg-Königstuhl, Königstuhl 12, 69117 Heidelberg, Germany \\ 2 Istituto Nazionale di Astrofisica (INAF), Osservatorio Astronomico di Torino, via Osservatorio 20, \\ 10025 Pino Torinese (TO), Italy \\ e-mail: [villata;raiteri]@to.astro.it
}

Received 5 December 2003 / Accepted 10 February 2004

\begin{abstract}
The long-term variability of the multiwavelength blazar emission can be interpreted in terms of orientation variations of a helical, inhomogeneous, non-thermally emitting jet, possibly caused by the orbital motion of the parent black hole in a binary system (Villata \& Raiteri 1999). The helical-jet model is here applied to explain the quasi-periodic radio-optical light curves and the broad-band spectral energy distributions (SEDs) of the BL Lac object AO 0235+16. Through a suitable choice of the model parameters, the helix rotation can well account for the periodicity of the main radio and optical outbursts and for the corresponding SED variability, while the interspersed minor radio events could be interpreted as due either to some local distortions of the helical structure or to other phenomena contributing to the source emission. In particular, the probable existence of flow instabilities provides a viable interpretation for the non-periodic features.
\end{abstract}

Key words. galaxies: active - galaxies: BL Lacertae objects: general - galaxies: BL Lacertae objects: individual: AO 0235+16 galaxies: jets - galaxies: nuclei - galaxies: quasars: general

\section{Introduction}

Blazars, namely BL Lacertae objects and flat-spectrum radio quasars, belong to the class of active galactic nuclei (AGNs). According to the unified model of AGNs (see e.g. Urry \& Padovani 1995), their emission is dominated by radiation produced in relativistic plasma jets, which are oriented at small angles with respect to the line of sight; the radiation is therefore strongly beamed towards the observer.

Details of the jet origin are still unknown, but their formation is thought to be triggered by the presence of a supermassive black hole surrounded by an accretion disc, possibly belonging to a binary black hole system (BBHS) hidden in the centre of the AGN. On the one hand, this scenario is suggested by the elliptical morphology of BL Lac host galaxies, which are believed to originate from merging phenomena between spirals, naturally leading to the formation of massive binary black holes (Begelman et al. 1980; Wilson \& Colbert 1995; Huges \& Blandford 2003). On the other hand, several items of observational evidence such as bending, misalignment, wiggling and precession of jets (Begelman et al. 1980;

Send offprint requests to: L. Ostorero, e-mail: 1.ostorero@lsw. uni-heidelberg.de
Camenzind \& Krockenberger 1992; Kaastra \& Roos 1992; Conway \& Wrobel 1995; Villata et al. 1998; Villata \& Raiteri 1999 and references therein; Abraham 2000; Britzen et al. 2001), often associated with knots superluminally moving along different-scale curved trajectories, as well as the periodicity discovered in the multiwavelength light curves of some of these sources, have in some cases been interpreted in terms of helical structures tightly related to BBHSs.

The helical-jet model proposed by Villata \& Raiteri (1999) describes how orientation variations of the different-frequency emitting parts of a curved jet with respect to the line of sight, possibly caused by the orbital motion of the parent black hole in a BBHS, can be the cause of the observed changes in the spectral energy distribution (SED) of a blazar. The emission from the jet is non-thermal: the relativistic electron population is responsible for producing both the low-energy (from radio to UV-X-rays) synchrotron radiation and the high-energy (up to $\gamma$-rays) radiation through inverse-Compton (IC) scattering of the same synchrotron photons (synchrotron-self-Compton process, hereafter SSC).

This model has already provided an interpretation of the huge X-ray spectral brightening of Mkn 501 (Villata \& Raiteri 1999), the low-energy SED variations of S4 0954+65 
(Raiteri et al. 1999), and the changes in the overall SEDs of S5 0716+71 (Ostorero et al. 2001) and ON 231 (Sobrito et al. 2001). The modelling of a constant helix rotation allows us now to simulate the long-term behaviour of the multifrequency light curves together with the SED time evolution. If the data sampling is good enough and the source emission exhibits periodic (or quasi-periodic) behaviour, one can apply the model and find the set of parameters which best reproduces the observed multifrequency data, thus providing both a test for the model and a description of the corresponding jet features.

In this paper the model, fully described in Sect. 2, is applied to the case of the BL Lac object AO 0235+16, whose radio (and optical) light curves have recently revealed a $\sim 5.7$ year quasiperiodicity (Raiteri et al. 2001). Model light curves and SEDs are compared with observational data in Sect. 3. Possible interpretations of minor, non-periodic events are presented in Sect. 4. A final discussion is given in Sect. 5.

\section{The helical-jet model}

The helical-jet model by Villata \& Raiteri (1999) foresees that, in a BBHS scenario, the emitting jet is bent because of the orbital motion and by the interaction of its magnetized plasma with the surrounding medium, twisting in a rotating helical structure.

Since in Villata \& Raiteri (1999) only the synchrotron emission was taken into account, we recall here the main formulae, adding those expressions relevant to the high-energy SSC emission (see also Raiteri et al. 2004).

Let us consider a helical jet with the helix axis taken as the $z$-axis of a 3-D reference frame. The helix has a pitch angle $\zeta$ and the line of sight forms an angle $\psi$ with the $z$-axis. The length of the helical jet path can be expressed as

$l(z)=\frac{z}{\cos \zeta}, \quad 0 \leq z \leq 1$,

and covers an azimuthal angle

$\varphi(z)=a z$

where $a$ is the total angle covered by the helix. The jet viewing angle at $z$ is

$\cos \theta(z)=\cos \psi \cos \zeta+\sin \psi \sin \zeta \cos (\phi-a z)$

where $\phi$ is the "rotation angle", i.e. the azimuthal difference between the line of sight and the initial direction of the helical path.

The jet geometry described above is the apparent shape of the plasma flow; the different light travel times of each jet region and the plasma velocity components due to the rotation of the helical structure have been taken into account.

The jet is assumed to be inhomogeneous, similarly to that of the model of Ghisellini \& Maraschi (1989; see also Ghisellini et al. 1985; Maraschi et al. 1992): each slice of it emits synchrotron radiation in a range of frequencies between $v_{\mathrm{s} \text {,min }}^{\prime}$ and $v_{\mathrm{s}, \max }^{\prime}$ (primed quantities refer to the plasma rest reference frame), which are supposed to decrease as power laws as the distance $l$ from the apex increases ${ }^{1}$ :

$$
\begin{aligned}
& v_{\mathrm{s}, \min }^{\prime}(l)=v_{\mathrm{s}, \min }^{\prime}(0)\left(1+\frac{l}{l_{1}}\right)^{-c_{1}}, \quad c_{1}>0, \\
& v_{\mathrm{s}, \max }^{\prime}(l)=v_{\mathrm{s}, \max }^{\prime}(0)\left(1+\frac{l}{l_{2}}\right)^{-c_{2}}, \quad c_{2}>0,
\end{aligned}
$$

where $v_{\mathrm{s}, \text { min }}^{\prime}(0)$ and $v_{\mathrm{s}, \text { max }}^{\prime}(0)$ are the values at $l=z=0, l_{1}$ and $l_{2}$ are length scales. In the following we assume that $v_{\mathrm{s}, \min }^{\prime}(0)=$ $v_{\mathrm{s}, \max }^{\prime}(0) \equiv v_{\mathrm{s}, 0}^{\prime}$. The jet also emits higher-energy radiation, via IC scattering of soft synchrotron photons off high-energy electrons (SSC process), in a range of frequencies

$v_{\mathrm{c}, \min }^{\prime}(l)=\frac{4}{3} \gamma_{\min }^{2} v_{\mathrm{s}, \min }^{\prime}(l)$,

$v_{\mathrm{c}, \max }^{\prime}(l)=\frac{4}{3} \gamma_{\max }^{2}(l) v_{\mathrm{s}, \max }^{\prime}(l)$,

where the electron Lorentz factor $\gamma$ is supposed to vary between $\gamma_{\min }=1$ and

$\gamma_{\max }(l)=\gamma_{0}\left(1+\frac{l}{l_{\gamma}}\right)^{-c_{\gamma}}, \quad c_{\gamma} \geq 0$,

being $\gamma_{0}=\gamma_{\max }(0)$.

Actually, in order to take the Klein-Nishina effect into account, an upper limit is set to $v_{\mathrm{c}, \max }^{\prime}(l)$, in the form

$v_{\mathrm{c}, \max }^{\prime}(l)=\gamma_{\max }(l) \min \left\{\frac{4}{3} \gamma_{\max }(l) v_{\mathrm{s}, \max }^{\prime}(l), \frac{m_{\mathrm{e}} c^{2}}{h}\right\}$.

The observed flux density at frequency $v$ is assumed to be

$F_{v}(v) \propto \delta^{3} v^{-\alpha_{0}}$,

where $\alpha_{0}$ is the power-law index of the local synchrotron spectrum (fixed to be $\alpha_{0}=0.5$ ) and

$\delta=[\Gamma(1-\beta \cos \theta)]^{-1}$

is the beaming or Doppler factor, $\beta$ being the bulk velocity of the emitting plasma in units of the speed of light, $\Gamma=(1-$ $\left.\beta^{2}\right)^{-1 / 2}$ the corresponding bulk Lorentz factor, and $\theta$ the angle between the velocity vector and the line of sight, which varies along the helical path as shown in Eq. (3), hence causing a dependence of $\delta$ on the $z$ coordinate.

Notice that the local viewing angle $\theta$ of the jet reaches a minimum when $\phi-a z$ is zero or a multiple of $360^{\circ}$, implying the maximization of the beaming effect for frequencies emitted from the corresponding jet segments.

\footnotetext{
${ }^{1}$ In the Ghisellini \& Maraschi (1989) model, what is parametrized and described with power laws are the physical quantities of the jet, on which the emitted frequencies depend. Since we are interested in Doppler factor variation effects (whatever the physical details can be), we prefer a more direct, phenomenological approach, in order to lower the parameter number. If needed, one can go back to the physical characteristics.
} 
By introducing an intrinsic flux-density dependence on $l$, the observed synchrotron and IC flux densities at frequency $v$ coming from a jet slice of thickness $\mathrm{d} l$ can be expressed as

$$
\begin{aligned}
\mathrm{d} F_{v, \mathrm{~s}}(v)= & K_{\mathrm{s}}\left(1+\frac{l}{l_{\mathrm{s}}}\right)^{-c_{\mathrm{s}}} \delta^{3}(l) v^{-\alpha_{0}} \mathrm{~d} l, & & c_{\mathrm{s}} \geq 0, \\
\mathrm{~d} F_{v, \mathrm{c}}(v)= & K_{\mathrm{c}}\left(1+\frac{l}{l_{\mathrm{c}}}\right)^{-c_{\mathrm{c}}} \delta^{3}(l) v^{-\alpha_{0}} & & \\
& \times \ln \frac{v_{\mathrm{s}, \text { max }}^{\prime}(l)}{v_{\mathrm{s}, \text { min }}^{\prime}(l)} \mathrm{d} l, & & c_{\mathrm{c}} \geq 0,
\end{aligned}
$$

where $l_{\mathrm{s}}$ and $l_{\mathrm{c}}$ are again length scales, and the constants $K_{\mathrm{s}}$ and $K_{\mathrm{c}}$ are considered to be independent of time, meaning that intrinsic variations of the flux are not allowed. The respective total flux densities at frequency $v$ coming from the whole jet are obtained by integrating along all the portions contributing to that observed frequency:

$$
\begin{aligned}
F_{v, \mathrm{~s}}(v)= & K_{\mathrm{s}} v^{-\alpha_{0}} \sum_{i} \int_{\Delta z_{\mathrm{s}, i}(v)}\left[1+\frac{l(z)}{l_{\mathrm{s}}}\right]^{-c_{\mathrm{s}}} \\
& \times \delta^{3}(z) \frac{\mathrm{d} l}{\mathrm{~d} z} \mathrm{~d} z \\
F_{v, \mathrm{c}}(v)= & K_{\mathrm{c}} v^{-\alpha_{0}} \sum_{i} \int_{\Delta z_{\mathrm{c}, i}(v)}\left[1+\frac{l(z)}{l_{\mathrm{c}}}\right]^{-c_{\mathrm{c}}} \\
& \times \delta^{3}(z) \frac{\mathrm{d} l}{\mathrm{~d} z} \ln \frac{v_{\mathrm{s}, \max }(z)}{v_{\mathrm{s}, \min }^{\prime}(z)} \mathrm{d} z
\end{aligned}
$$

where $\mathrm{d} l / \mathrm{d} z=1 / \cos \zeta$, and $\Delta z_{\mathrm{s}, \mathrm{c} ; i}(v)$ is the $z$ interval corresponding to the $i$ th jet segment emitting radiation at frequencies observed as $v$ along the line of sight, i.e. where the condition

$\delta(z) v_{\mathrm{s}, \mathrm{c} ; \min }^{\prime}(z) \leq v \leq \delta(z) v_{\mathrm{s}, \mathrm{c} ; \max }^{\prime}(z)$

is satisfied. An explanation of the logarithmic term in Eqs. (13) and (15) can be found in Ghisellini \& Maraschi (1989).

The total flux density at frequency $v$ is finally obtained by summing the synchrotron and IC contributions.

If the helix rotates, e.g. because of the orbital motion of the parent black hole in a BBHS, the $\phi$ angle, and hence $\theta$ (Eq. (3)), vary with time; this consequently makes the Doppler factor $\delta$ (Eq. (11)) of each emitting portion $\mathrm{d} l$ of the jet timedependent, thus causing a temporal evolution of the observed flux density $F_{v}(v)$, even if the jet emission has not intrinsically changed at all.

\section{Modelling the periodic long-term behaviour of AO 0235+16}

The recently discovered periodic behaviour of radio (and optical) light curves of AO $0235+16$ (Raiteri et al. 2001) and the wealth of historical spectral coverage of this source suggested to us the advisability of applying the rotating helical-jet model, intrinsically periodic, to model the long-term trend of its multifrequency light curves and to describe the corresponding broad-band SED time evolution (preliminary results of this work have already been published in Ostorero et al. 2003a and Ostorero et al. 2003b).

With this purpose, we assembled the historical light curves and composed the multi-epoch SED of AO $0235+16$, by collecting data at all available frequencies from the literature and homogenizing them through suitable corrections for extinction effects. In particular, optical and infrared magnitudes were converted into fluxes using the absolute calibrations of Bessel (1979) for standard UBVKF and Cousins' RI filters, Wamsteker (1981) for standard $J H L$ bands, and Allen (1973) for standard RIN filters; infrared-to-ultraviolet dereddening was computed adopting the extinction laws of Rieke \& Lebofsky (1985) and Cardelli et al. (1989), assuming a Galactic extinction $A_{B}=0.341 \mathrm{mag}$ (from NED). In the X-ray band, absorbed fluxes were corrected for total absorption fading using the XSPEC procedure.

\subsection{The radio-optical light curves}

The most time-extended and best-sampled light curves of the source are the optical ones (see Fig. 1a), and the radio ones at $14.5 \mathrm{GHz}$ (Fig. 1b), $8.0 \mathrm{GHz}$ (Fig. 1c) and $4.8 \mathrm{GHz}$ (Fig. 1d) from the University of Michigan Radio Astronomy Observatory (UMRAO). All these curves exhibit strong variability: many large-amplitude outbursts lasting from several months to a few years witness an intense source activity. From the long-term time analysis of the best-sampled light curve at $8.0 \mathrm{GHz}$, Raiteri et al. (2001) found the signature of a quasiperiodic behaviour, also recognizable in the other radio bands: the major outbursts repeat quasi-regularly every $\sim 5.7$ years, many of them accompanied by simultaneous or slightly preceding optical ones. A visual inspection of the curves in the left panels of Fig. 1 reveals that the optical events are however sharper, more pronounced, and sometimes more frequent than the radio ones. Indeed, Takalo et al. (1998) invoked the existence of two different optical emission mechanisms: one also responsible for the radio events (likely synchrotron processes in the relativistic jet), and the other not (probable microlensing effect of a foreground galaxy on radiation coming from either the accretion disc or other jet regions). Moreover, the radio outbursts have decreasing amplitude with increasing wavelength, and flux variations at the lower frequencies lag behind those at the higher frequencies (see the cross-correlation analysis performed by Raiteri et al. 2001); other minor outbursts are present in the radio light curves beside the periodic events. Finally, all outbursts and flaring events seem to be superimposed onto a slowly decreasing "base level" flux (see Sect. 3.2 for details).

Confirmation of this behaviour comes from the lowerenergy radio light curves, notwithstanding their poor sampling. The delayed counterparts of both the 1982 periodic radio outburst and the 1980-81 non-periodic event are likely represented by the pairs of peaking structures sequentially appearing in the curves at $1.4 \mathrm{GHz}$ (Fig. 1e; dots) and $880 \mathrm{MHz}$ (Fig. 1f) from the Green Bank telescope, at $1.5 \mathrm{GHz}$ (Fig. 1e; crosses) from the NRAO, and at $430 \mathrm{MHz}$ (Fig. 1g) from the Arecibo telescope. Furthermore, the outburst peak flux seems to decrease with increasing wavelength more rapidly in the case of 

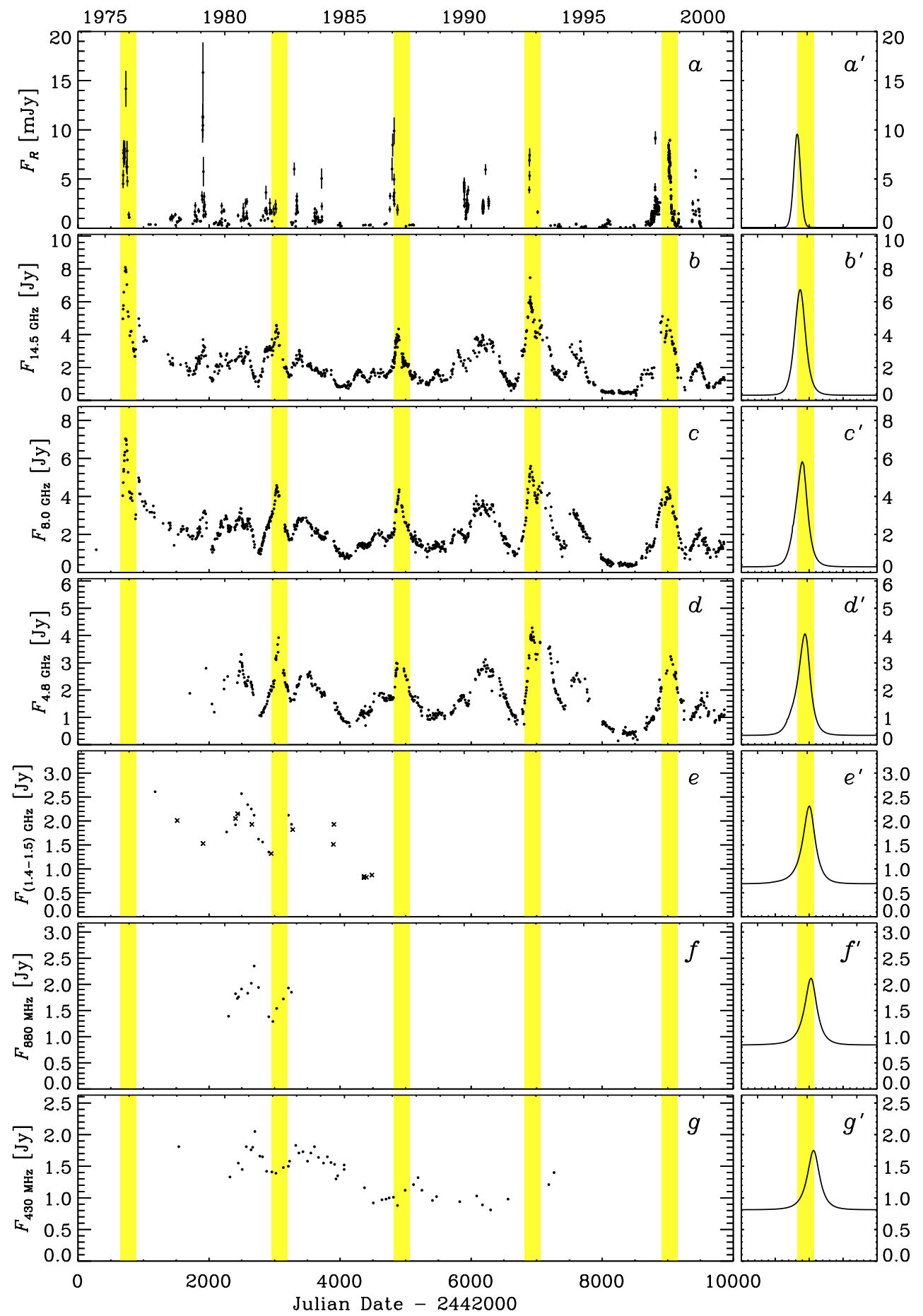

Fig. 1. Left panels: observed optical (mJy) and radio (Jy) light curves of AO $0235+16$. a) $R$ optical band ( $R$ band data, and $B$ band data before JD = 2449000 converted into $R$ by adopting a mean colour index $\langle B-R\rangle=1.65 \pm 0.16$; see Raiteri et al. 2001 for details); b)-d) 14.5 , 8.0, $4.8 \mathrm{GHz}$ radio bands (UMRAO); e) $1.4 \mathrm{GHz}$ (dots; Green Bank telescope) and $1.5 \mathrm{GHz}$ (crosses; NRAO) radio bands; the data have been plotted together to better define the light curve features; f) $880 \mathrm{MHz}$ radio band (Green Bank telescope); g) $430 \mathrm{MHz}$ radio band (Arecibo telescope). These light curves are based on data from: Owen et al. (1978), Owen et al. (1980), Ulvestad et al. (1981), Perley (1982), Briggs (1983), Landau et al. (1983), Ulvestad et al. (1983), Altschuler et al. (1984), Ulvestad \& Johnston (1984), Aller et al. (1985), Rudnick et al. (1985), Brown et al. (1989), Salgado et al. (1999), Raiteri et al. (2001) and references therein. Right panels: Helical-jet modelling of the typical multifrequency outbursts shown in the left panels (see the text for a detailed description of the model parameters relevant to curves $\mathbf{a}^{\prime}$ )- $\mathbf{g}^{\prime}$ ): as the wavelength increases, the outbursts appear to be more and more delayed and to have smaller amplitude and longer duration, in agreement with observations. Grey (yellow in the electronic version) strips highlight here the time interval between the peak of the modelled optical outburst and the maximum of the associated lowest-energy radio event. The same strips have been drawn on the observed light curves of the left panels, in correspondence to the periodic outbursts, even if in more than one case some counterparts are poorly sampled or missing entirely. 
the periodic events than in the case of the non-periodic ones. Thus, while at high radio frequencies the periodic outbursts dominate the non-periodic ones, at low energies the opposite seems to occur; this behaviour implies different radio broadband spectral indices for the periodic and non-periodic components. Moreover, the light curve at $430 \mathrm{MHz}$ (Fig. 1g) also exhibits the presence of a decreasing "base level" flux, on which the peaks corresponding to the periodic trend fade almost completely away. Notice also that the "base level" flux seems not to decrease with decreasing radio frequency (a similar behaviour was recognized in the BL Lac object S5 0716+71; see Raiteri et al. 2003).

As anticipated in the previous section, the rotation of an inhomogeneous helical jet may account for the periodic behaviour of multiwavelength light curves. As the helix rotates, different-frequency emitting portions of the jet approach the line of sight, giving rise to outbursts observed at lower and lower energies as long as the rotation angle $(\phi)$ increases: this phenomenon comes from both the jet inhomogeneity and the beaming effect, as will be discussed in the following.

The radio-optical light curve modelling was performed assuming a period of 2069 days (see Raiteri et al. 2001) for the constant helix rotation, and by fixing $\phi=0$ at JD = 2451032.6341 , corresponding to the 1998 outburst observed peak ( $R$ band).

The strongest constraints to be taken into account in setting the model parameters are the observed outburst amplitudes, their durations, and the shortness of the delay of the radio events with respect to the optical ones, which strongly depend on the inhomogeneity of the first part of the jet.

This inhomogeneity was modelled to meet the observational constraints by replacing the general single power-law trends for the emitted frequencies of the helical-jet model (Eqs. (4) and (5)) with more complex laws.

In particular, the power law describing the minimum synchrotron frequency emitted along the jet path, $v_{\mathrm{s}, \min }^{\prime}(l)$, was replaced by a combination of power laws for $l \leq l_{\text {break }}$ :

$$
\begin{aligned}
v_{\mathrm{s}, \min }^{\prime}(l)= & v_{\mathrm{s}, 0, \mathrm{I}}^{\prime}\left(1+\frac{l}{l_{1, \mathrm{I}}}\right)^{-c_{1, \mathrm{I}}} \\
& +v_{\mathrm{s}, 0, \mathrm{II}}^{\prime}\left(1+\frac{l}{l_{1, \mathrm{II}}}\right)^{-c_{1, \mathrm{II}}}, \quad c_{1, \mathrm{I}}, c_{1, \mathrm{II}}>0,
\end{aligned}
$$

and with the tangent to $\log v_{\mathrm{s}, \min }^{\prime}(l)$ in $l=l_{\text {break }}$, for $l>l_{\text {break }}$, while the $v_{\mathrm{s}, \max }^{\prime}(l)$ relation is simply a linear combination of power laws like that of Eq. (5):

$$
\begin{aligned}
v_{\mathrm{s}, \text { max }}^{\prime}(l)= & v_{\mathrm{s}, 0, \mathrm{I}}^{\prime}\left(1+\frac{l}{l_{2, \mathrm{I}}}\right)^{-c_{2, \mathrm{I}}} \\
& +v_{\mathrm{s}, 0, \mathrm{II}}^{\prime}\left(1+\frac{l}{l_{2, \mathrm{II}}}\right)^{-c_{2, \mathrm{II}}}, \quad c_{2, \mathrm{I}}, c_{2, \mathrm{II}}>0 .
\end{aligned}
$$

Both relations are represented by the continuous (blue) curves of Fig. 2 (the parameter values are given in Table 1, Col. A).

The minimum emitted frequency law is very steep at first, implying the emission of frequencies down to radio bands from the immediate vicinity of the apex of the emitting jet. This allows the observed optical and radio outbursts to follow one another with short time delays, as the contiguous jet slices mainly

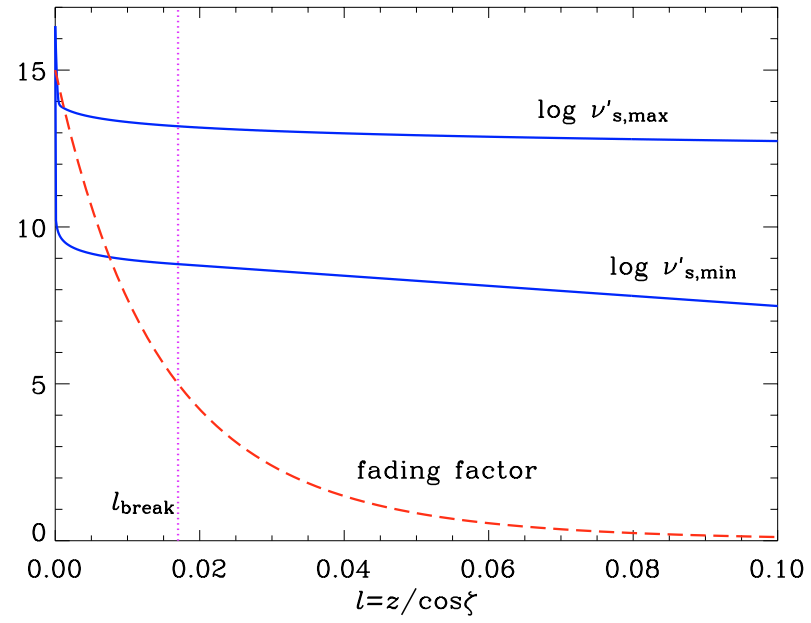

Fig. 2. Solid (blue) curves represent the minimum and maximum synchrotron frequencies emitted by the modelled jet of AO $0235+16$, as a function of the helical path coordinate $l$. Note the steepness of the $v_{\mathrm{s}, \min }^{\prime}(l)$ law in the first jet slice and its more slowly decreasing trend right after the apex region. The vertical, dotted (purple) line represents the point $l_{\text {break }}=0.017$ where the law has been replaced by a linear decreasing trend. The law parameters are given in Col. $A$ of Table 1. The dashed (red) curve shows the decrease, along the jet, of the intrinsic fading factor: it dramatically reduces the emission well before the first tenth of the path, making the jet region far from the apex contribute marginally to the whole emission. Only the portion of the path between $l=0$ and $l=0.1$ has been represented here. For graphic convenience, the fading factor has been normalized to 15 .

producing them become more aligned with the line of sight during the helix rotation. Then, the linear decrease of $\log v_{\mathrm{s}, \min }^{\prime}$ makes the emission of the observed very soft radio photons possible far from the jet apex, before the intrinsic flux-density dependence on $l$, represented by the "fading factor" $\left(1+l / l_{\mathrm{s}}\right)^{-c_{\mathrm{s}}}$ (see Eq. (11)), strongly reduces the jet emission (see the dashed red curve of Fig. 2).

The results of the helical-jet modelling of the light curves of Figs. $1 \mathrm{a}-\mathrm{g}$ are displayed in the right panels of Figs. $1 \mathrm{a}^{\prime}-\mathrm{g}^{\prime}$. Since the model light curves are exactly periodic, only snapshots including outbursts are shown for a visual comparison between the modelled and observed features. Grey (yellow in the electronic version) stripes, defined as the time interval ( 242 days) between the optical theoretical peak and the lowest-frequency radio peak, guide the eye through the periodic outbursts in different energy bands. Besides the periodic recurrence, also the typical shape of the main observed optical and radio outbursts, the decrease of the peak flux with decreasing radio frequency, and the optical-to-radio flux ratio are well described by the model. The quasi-simultaneity of optical and radio outbursts is also well reproduced: radio delays start from about a couple of months in the case of the shortest wavelengths, consistent with the results of the cross-correlation analysis performed by Raiteri et al. (2001), and gradually increase up to $\sim 8$ months, as one moves towards the lowest frequencies.

A complete summary of the parameters describing the geometrical and physical properties of the modelled AO $0235+16$ jet is given in Table 1: both sets $A$ and $B$ (see Sect. 3.2 for 
Table 1. Helical-jet model input parameters relevant to light curve and SED evolution modelling of AO $0235+16$. Column A: light curves of Fig. 1 (right panels) and Fig. 5a, and SED time evolution of Fig. 3. Column $B$ : SED time evolution of Fig. 4. Values differing in the two models are written in boldface.

\begin{tabular}{|c|c|c|}
\hline Jet geometry & $A$ & $B$ \\
\hline$\zeta$ & $20^{\circ}$ & $20^{\circ}$ \\
\hline$\psi$ & $15^{\circ}$ & $15^{\circ}$ \\
\hline$a$ & $360^{\circ}$ & $360^{\circ}$ \\
\hline Jet physics & $A$ & $B$ \\
\hline$\Gamma$ & 10 & 10 \\
\hline$\gamma_{\min }$ & 1 & 1 \\
\hline$\gamma_{0}$ & $1.995 \times 10^{5}$ & $6.310 \times 10^{3}$ \\
\hline$c_{\gamma}$ & 0 & 0 \\
\hline Synchrotron radiation & $A$ & $B$ \\
\hline$\alpha_{0}$ & 0.5 & 0.5 \\
\hline$v_{\mathrm{s}, 0, \mathrm{I}}^{\prime}$ & $10^{14}$ & $10^{14}$ \\
\hline$v_{\mathrm{s}, 0, \mathrm{II}}^{\prime}$ & $1.259 \times 10^{14}$ & $2.512 \times 10^{16}$ \\
\hline$l_{\text {break }}$ & 0.017 & 0.017 \\
\hline$l_{1, \mathrm{I}}=l_{1, \mathrm{II}}$ & $10^{-10}$ & $10^{-10}$ \\
\hline$l_{2, \mathrm{I}}=l_{2, \mathrm{II}}$ & $10^{-3}$ & $10^{-3}$ \\
\hline$c_{1, \mathrm{I}}=c_{2, \mathrm{I}}$ & 0.63 & 0.63 \\
\hline$c_{1, \mathrm{II}}=c_{2, \mathrm{II}}$ & 9.45 & 9.45 \\
\hline$l_{\mathrm{s}}$ & 0.1 & 0.1 \\
\hline$c_{\mathrm{s}}$ & 7 & 7 \\
\hline$K_{\mathrm{s}}$ & $5.012 \times 10^{4}$ & $5.012 \times 10^{4}$ \\
\hline IC radiation & $A$ & $B$ \\
\hline$\overline{l_{\mathrm{c}}}$ & 0.1 & 0.1 \\
\hline$c_{\mathrm{c}}$ & 7 & 7 \\
\hline$K_{\mathrm{c}}$ & 5.012 & 5.012 \\
\hline
\end{tabular}

details) give rise to the same radio-to-optical light curves. The emerging picture for the jet of $\mathrm{AO} 0235+16$ is a rotating helical structure with a pitch angle $\zeta=20^{\circ}$ and the axis oriented at an angle $\psi=15^{\circ}$ with respect to the line of sight, implying for each jet slice a maximum alignment of $5^{\circ}$. The plasma flows along the helix with a bulk Lorentz factor $\Gamma=10$, and emits synchrotron and IC radiation, all photons mainly coming from a jet region close to the apex.

In general, the parameter values reported in Table 1 represent the almost unique set of parameters that can strictly account for the multifrequency light curve (and SED, see below) behaviour, due to the strong constraints imposed by the exceptional dataset. However, some parameters, namely the jet geometry, $\Gamma$, and the IC parameters, are less constrained for two independent reasons. For the jet geometry and $\Gamma$, it is the Doppler factor variations that are strictly constrained by the data, so that other combinations of $\Gamma$ and angles can work as well. On the other hand, the high-energy part of the SED is poorly sampled (see next subsection) and does not allow one to identify a unique choice of parameters. In conclusion, the phenomenological parameters (and the corresponding physical quantities, see footnote 1) we have derived here to match the data can represent the "most reasonable" jet features, but not the sole solution.

\subsection{The SED time evolution}

The BL Lac object AO $0235+16$ has been monitored in a wide range of frequencies since the 1970s. Radio observations were performed by many antennas, starting from about $100 \mathrm{MHz}$ up to $300 \mathrm{GHz}$; infrared space and ground data are available from submillimetric (far-IR) down to $\mu \mathrm{m}$ wavelengths (nearIR); optical UBVRI bands have been intensively monitored by many telescopes all around the world, while only one ultraviolet upper limit by IUE is available. AO $0235+16$ was also detected in the X-ray band by several space observatories operating within the range $0.5-60 \mathrm{keV}$ (Einstein, EXOSAT, ROSAT, ASCA, RXTE) starting from 1978. The $\gamma$-ray emission was investigated through the instruments onboard the Compton Gamma-Ray Observatory (CGRO): COMPTEL probed the low-medium energy $\gamma$-ray range of the source spectrum during CGRO Cycle 4 (1994-1995), and only set upper limits to the flux in the interval $0.75-30 \mathrm{MeV}$; EGRET explored the higher-energy $\gamma$-ray radiation $(30 \mathrm{MeV}-20 \mathrm{GeV})$ with six pointings between 1992 and 1997, and with high confidence identified $\mathrm{AO} 0235+16$ as a powerful $\gamma$-ray emitter, providing two detections and four upper limits.

Figures 3 and 4 show the multi-epoch broad-band SEDs of the source, composed by collecting literature data at all available wavelengths: the historical variation ranges of the emission from radio to optical bands are represented together with all the X-ray and $\gamma$-ray data. The low-energy part of the SED exhibits a variability which becomes less pronounced moving from optical bands towards soft radio frequencies, as is also evident from the previously discussed light curves of Fig. 1. A variability range and a clear broad-band behaviour cannot instead be easily identified for the high-energy spectral component, because of the poor sampling of the X-ray and $\gamma$-ray light curves, and the uncertainties in data analysis, as detailed in the following.

In the X-ray energy domain, the source reveals a remarkable variability: the $1 \mathrm{keV}$ flux spans less than one order of magnitude, but the spectral slope shows an intriguing alternation of steepening and hardening in the energy range $0.5-10 \mathrm{keV}$, the spectral index $\alpha\left(F_{v} \propto v^{-\alpha}\right)$ ranging from 0.41 to 2.25 . In particular, the spectrum seems to be steeper when the source is in a brighter state, as already noticed by Madejski et al. (1996), who analysed the spectral changes of the source from the 1993 detections by ROSAT to those in 1994 by ASCA: such behaviour, in disagreement with a simple synchrotron cooling model for the X-ray emission, could reveal that in AO $0235+16$ two different spectral components overlap in the X-ray band, the drop in intensity of the softer one uncovering the harder one. However, the softer component, which could be the tail of the synchrotron spectrum, is not so clearly identified, because of the large uncertainties in the X-ray spectral indices (see the X-ray butterflies in Figs. 3 


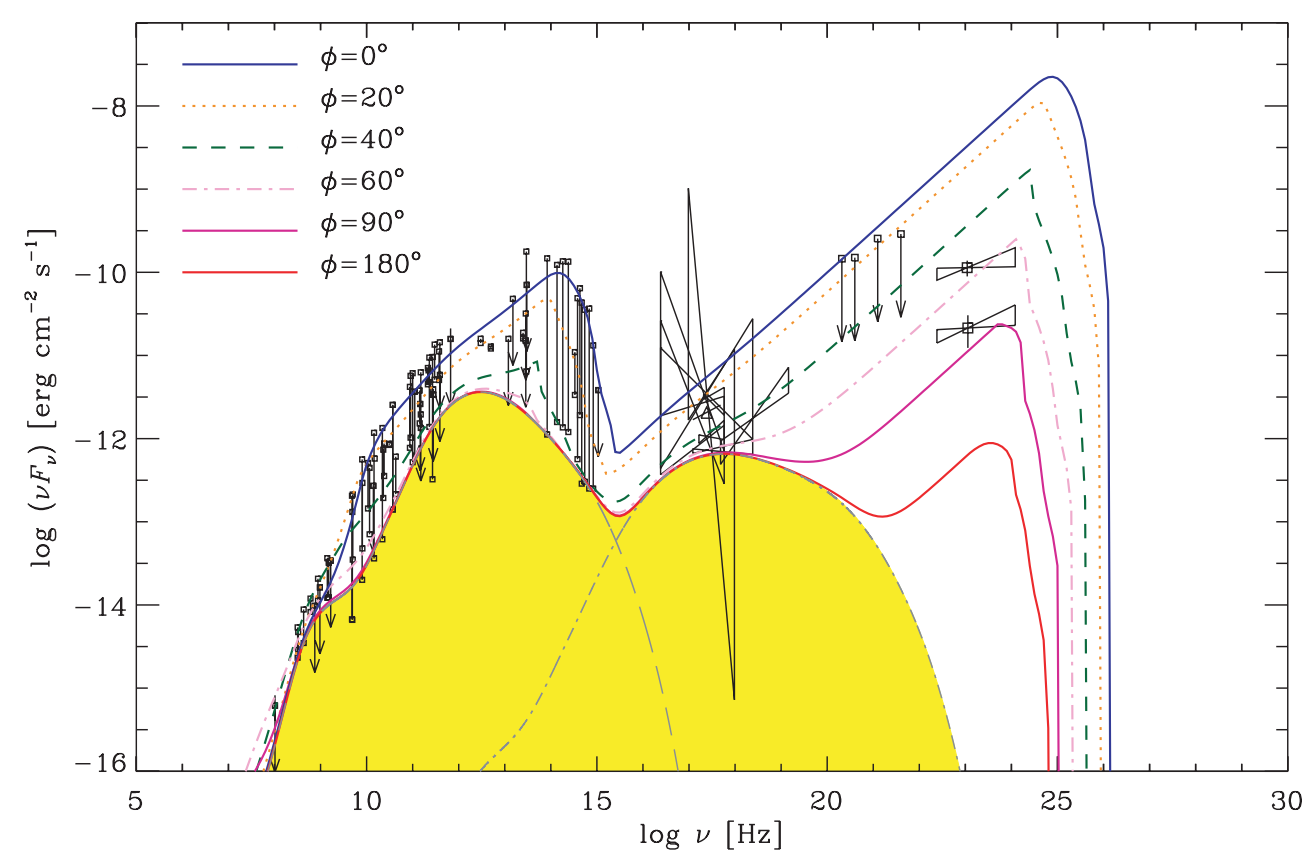

Fig. 3. Multi-epoch SED of AO $0235+16$. At radio, infrared, optical and ultraviolet frequencies, squares represent historical variation ranges when joined by vertical bars and single sporadic data otherwise; data are from: Condon \& Jauncey (1974), Ryle et al. (1975), Altschuler \& Wardle (1976), Rieke et al. (1976), O'Dell et al. (1977), Rieke et al. (1977), O'Dell et al. (1978a), O'Dell et al. (1978b), Owen et al. (1978), Condon et al. (1979), Epstein et al. (1980), Landau et al. (1980), Owen et al. (1980), Weiler \& Johnston (1980), Bååth et al. (1981), Jones et al. (1981), Altschuler (1982), Ennis et al. (1982), Impey et al. (1982), Altschuler (1983), Briggs (1983), Landau et al. (1983), Seielstad et al. (1983), Sitko et al. (1983), Ulvestad et al. (1983), Altschuler et al. (1984), Cotton et al. (1984), Cruz-Gonzales \& Hucra (1984), Gear et al. (1984), Holmes et al. (1984), Impey et al. (1984), Ulvestad \& Johnston (1984), Gear et al. (1985), Moles et al. (1985), Rudnick et al. (1985), Sitko et al. (1985), Brindle et al. (1986), Gear et al. (1986), Jones et al. (1986), Edelson (1987), Haddock \& Aller (1987), Salonen et al. (1987), Smith et al. (1987), Fugmann \& Meisenheimer (1988), Impey \& Neugebauer (1988), Steppe et al. (1988), Wardle \& Roberts (1988), Webb et al. (1988), Brown et al. (1989), Vetukhnovskaya (1989), Impey \& Tapia (1990), Mead et al. (1990), Simonetti \& Cordes (1990), Gregory \& Condon (1991), Sitko \& Sitko (1991), Edelson et al. (1992), Quirrenbach et al. (1992), Takalo et al. (1992), Teräsranta et al. (1992), Gear (1993), Krichbaum et al. (1993), Steppe et al. (1993), Bloom et al. (1994), Gear et al. (1994), Ghosh et al. (1994), Lichtfield et al. (1994), Tornikoski et al. (1996), Cotton et al. (1997), Reuter et al. (1997), Shen et al. (1997), Nartallo et al. (1998), Takalo et al. (1998), Salgado et al. (1999), Raiteri et al. (2001) and references therein. At high-energies, all data have been drawn: X-ray data (Einstein, EXOSAT, ROSAT, ASCA, RXTE) are from Worrall \& Wilkes (1990), Ghosh \& Soundararajaperumal (1995), Madejski et al. (1996), Comastri et al. (1997), Webb et al. (2000), and the TARTARUS database; $\gamma$-ray data (COMPTEL and EGRET) are from Schönfelder et al. (2000), Hartman et al. (1999), and Raiteri et al. (2001). The shaded (yellow) area shows the "base level" flux (synchrotron component: dashed line; IC component: dashed-dotted line). Different style (and colour) lines represent the SED evolution modelled with the helix rotation, i.e. with different values of the rotation angle $\phi$ (see the legend in the figure), on the hypothesis that the whole X-ray emission comes from IC scattering of synchrotron photons off jet electrons. Model parameters are shown in Col. $A$ of Table 1.

and 4). The main problem in the data analysis concerns indeed the estimate of the soft X-ray absorption. A model with a power-law continuum and absorption usually fits well the spectrum of $\mathrm{AO} 0235+16$, and the best fit is obtained by letting the hydrogen column density parameter $N_{\mathrm{H}}$ free; in this case, however, $N_{\mathrm{H}}$ is larger than the standard value. Alternatively, one can keep $N_{\mathrm{H}}$ fixed, obtaining smaller uncertainties on the spectral indices but a worse fit. From spectral studies, Madejski et al. (1996) concluded that the excess absorption effect is likely due to an intervening galaxy located at $z=0.524$, and performed a more accurate estimate of the spectral indices by introducing the absorber at $z=0.524$ in the analysis. However, the ambiguity in the X-ray spectral behaviour of the source is not completely solved, and the debate on the origin of X-rays in AO 0235+16 still remains open.

At $\gamma$-ray energies, the two clear detections by EGRET in 1994 and 1998 are not enough to constrain the source variability range. Only the 1994 signal is sufficiently strong to produce a useful spectrum, with a photon index $\gamma=1.88 \pm 0.11$ (Mukherjee et al. 1997; Hartman, private communication), even if a similar index $\gamma=1.85 \pm 0.12$ was derived from the sum of the observations of all viewing periods from Cycle 1 up to Cycle 4 (P1234; see Hartman et al. 1999).

As already noticed for the light curves of Fig. 1, the multiwavelength flux changes appear to be superimposed on a possible slowly variable "base level" emission, the deepest minimum of which is represented by the shaded (yellow) area of Figs. 3 and 4: its low-energy part was determined by extracting the most reliable historical minima from well-sampled radio, infrared, and optical light curves, and by fitting the corresponding SED with a cubic spline interpolation, while the high-energy part was analytically modelled according to the SSC theory, under the assumption that electron energies vary from $\gamma=1$ to $\gamma=10^{5}$. 


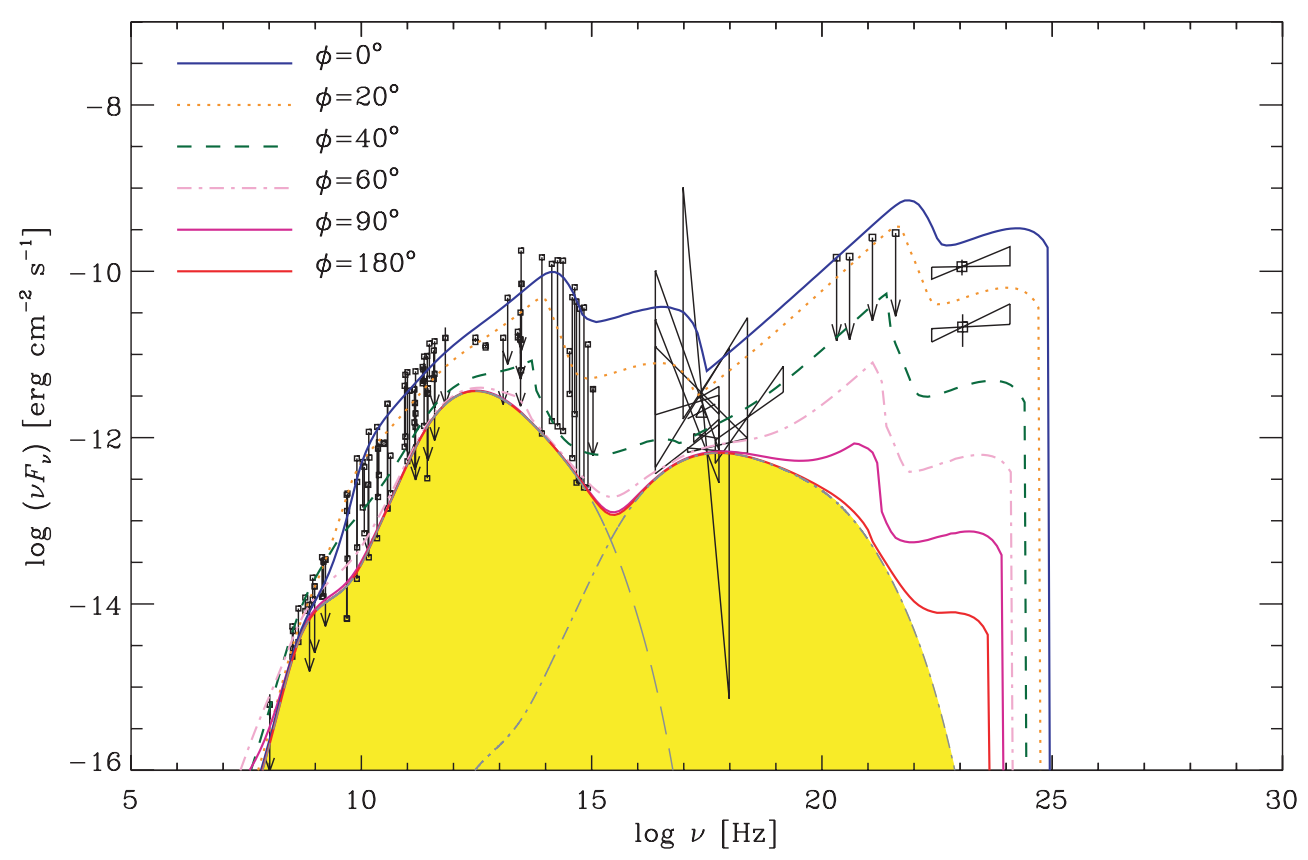

Fig. 4. Multi-epoch SED of AO 0235+16. Historical data and "base level" flux are the same of the SED in Fig. 3. Helical-jet model fits have been performed here by supposing the existence of a synchrotron contribution to the soft X-ray emission, while the hard X-rays still have an IC origin. Model parameters are shown in Col. $B$ of Table 1.

The long-term behaviour of the observed SED variability upon the "base level" was modelled as the result of the rotation of the steadily emitting helical jet previously described. In particular, the low-energy SED evolution is consistent with the radio-optical light curve modelling, while for the high-energy part two independent cases were considered to take the observation constraints into account: the soft X-ray emission is, in the first case, IC radiation, while in the second case it also has a synchrotron contribution, which is found to dominate for small $\left(\$ 20^{\circ}\right)$ rotation angles. Different style (and colour) curves in Figs. 3 and 4 show the model predictions for the SED time evolution in the two different hypotheses, as described in more detail below.

The time evolution of the low-energy branch of the spectrum, mainly resulting from synchrotron emission of the leptonic jet population, is common to both cases. As shown in Figs. 3 and 4, the kinematic evolution of the helical jet described in Sect. 3.1 can well account for the SED changes corresponding to the radio-optical light curve periodic behaviour (Fig. 1): as the $\phi$ angle increases with the rotation and the first, strongly emitting portion of the jet becomes more and more misaligned, the synchrotron peak slowly moves towards lower energies, finally plunging into the "base level" SED.

The high-energy part of the SED was studied taking the historical X-ray spectral properties of the source into account. As previously mentioned, the shape of the X-ray spectrum is extremely variable, being alternately steep and flat, and thus raising the controversial issue of the origin of the source $\mathrm{X}$-ray emission. Depending on the value of $\alpha$, it could either be synchrotron radiation produced by high-energy electrons in the jet, or come from IC scattering of soft synchrotron photons (SSC) or external radiation (EC) off jet electrons, or be the result of a composition of synchrotron and IC effects.
We hence simulated the high-energy SED time evolution in two different cases.

In the first (Fig. 3), we assumed that the whole X-ray jet emission comes from IC scattering of softer synchrotron photons off jet energetic electrons ( $\gamma_{0} \approx 2 \times 10^{5}$, see Eq. (8)).

The X-ray flux variability and the constancy of the relevant spectral slope, exhibited by the modelled emission as the rotation goes on, reflect the radio SED evolution, while the $\gamma$-ray SED fluctuations correspond to those in the infrared-optical band. The parameter setting is shown in Col. A of Table 1.

In the second case (Fig. 4), we supposed the existence of a synchrotron contribution to the soft X-ray emission to justify the steepest spectra, like those observed by the Einstein Observatory in August 1980 and by ROSAT in July-August 1993; in contrast, hard X-rays were still assumed to come from IC scattering of softer photons. The modelled jet is now characterized by the production of synchrotron radiation up to frequencies much higher than in the previous case $\left(\sim 10^{16} \mathrm{~Hz}\right)$ in the very first portion of the emitting jet. The consequence is the appearance of a synchrotron "bump" in the SED of Fig. 4 in the soft X-ray band, well visible as long as the jet apex region is oriented at small angles $\left(\phi \lesssim 20^{\circ}\right)$ to the line of sight. Moreover, the soft X-ray part of the SED becomes much more variable in both flux and slope, thus matching better the observed spectra. The $\gamma$-ray modelled emission also exhibits remarkable new features. It is the result of IC processing of a much wider range of frequencies and seems to better match observations with relatively low electron energies $\left(\gamma_{0} \approx 6 \times 10^{3}\right)$. The first SED peak occurs in the $\mathrm{MeV}$ band and corresponds to the infrared one, while the second peak lies in the $\mathrm{GeV}$ region and reflects the synchrotron X-ray bump, which thus would be the source of the seed photons for the EGRET 

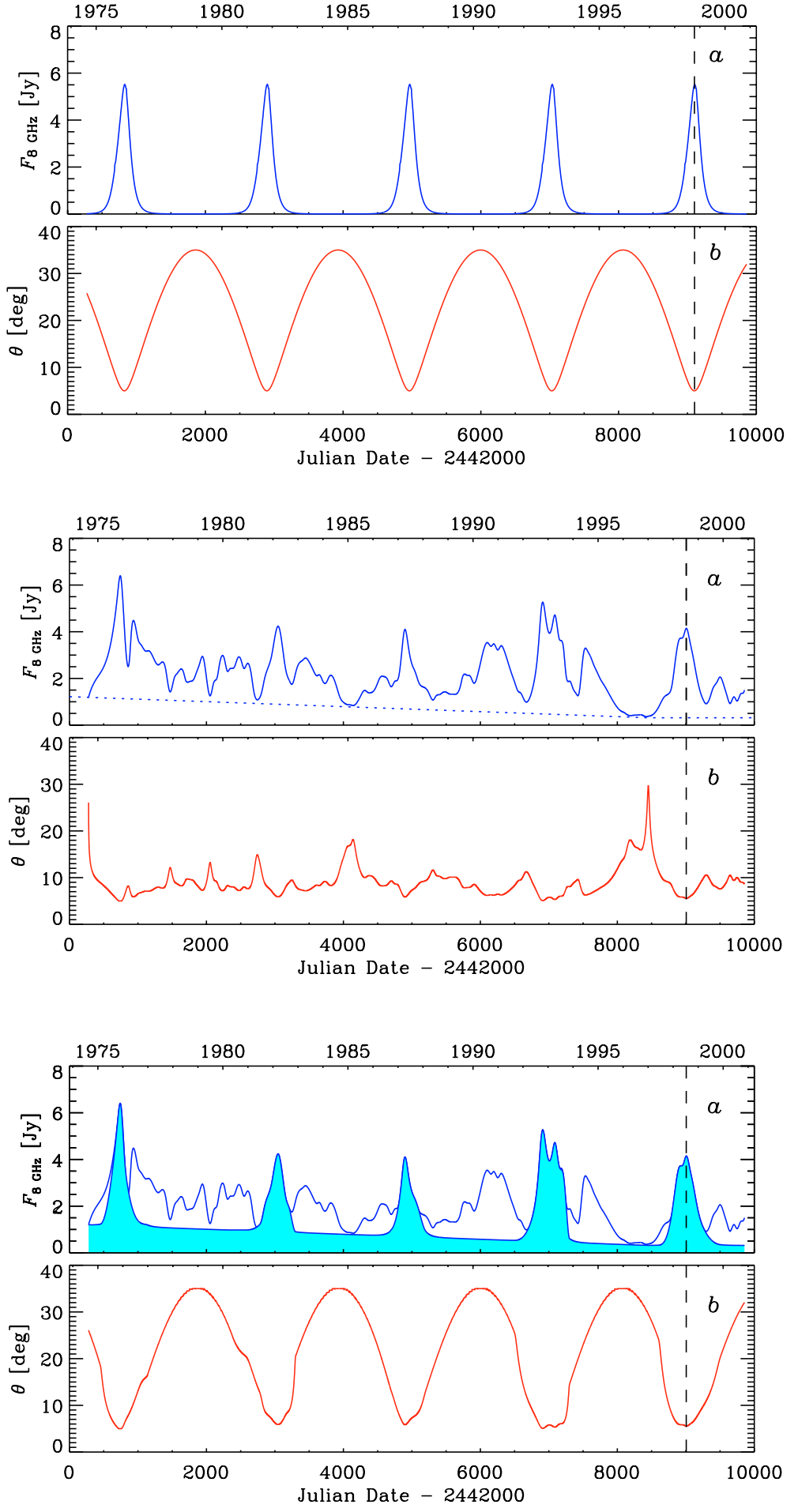

Fig. 5. a) Helical-jet modelling of the $8 \mathrm{GHz}$ light curve. b) Time evolution, with the helix rotation, of the viewing angle $\theta$ of the jet region emitting radiation observed at $8 \mathrm{GHz}$. Minima of the viewing angle $\theta$ correspond to maxima of the flux, as marked by the vertical dashed line. Only jet emission has been considered here.

Fig. 6. a) Cubic spline interpolation through the binned (50 days/bin) $8 \mathrm{GHz}$ light curve (solid line) and "base level" flux (dotted line). b) Model time evolution of the viewing angle of the $8 \mathrm{GHz}$ jet emitting region, derived from the light curve of panel a) on the hypothesis that minor, non-periodic outbursts are due to some distortions of the helical structure.

Fig. 7. a) The same as Fig. 5a, with the periodic component highlighted by shading. b) Time evolution of the viewing angle corresponding to the shaded light curve of panel a). detections. A complete description of the model parameters is given in Col. $B$ of Table 1 .

\section{Modelling the non-periodic events: The $8 \mathrm{GHz}$ light curve}

With the aim of investigating the variability of the source emission in greater detail, we attempted a more accurate comparison between model and observations for the best-sampled radio light curve at $8 \mathrm{GHz}$.
The model predicts that, as the helix rotates, the viewing angle of the $8 \mathrm{GHz}$ jet emitting region varies periodically, as shown in Fig. 5b, implying the periodic behaviour of the light curve displayed in Fig. 5a.

In order to make the comparison between model and data easier, we removed the short-term flaring from the observed curve of Fig. 1c by deriving a binned light curve with a bin size of 50 days, and by fitting it with a cubic spline interpolation (Press et al. 1992), as shown in Fig. 6a. The minor, nonperiodic outbursts interspersed among the periodic major ones 

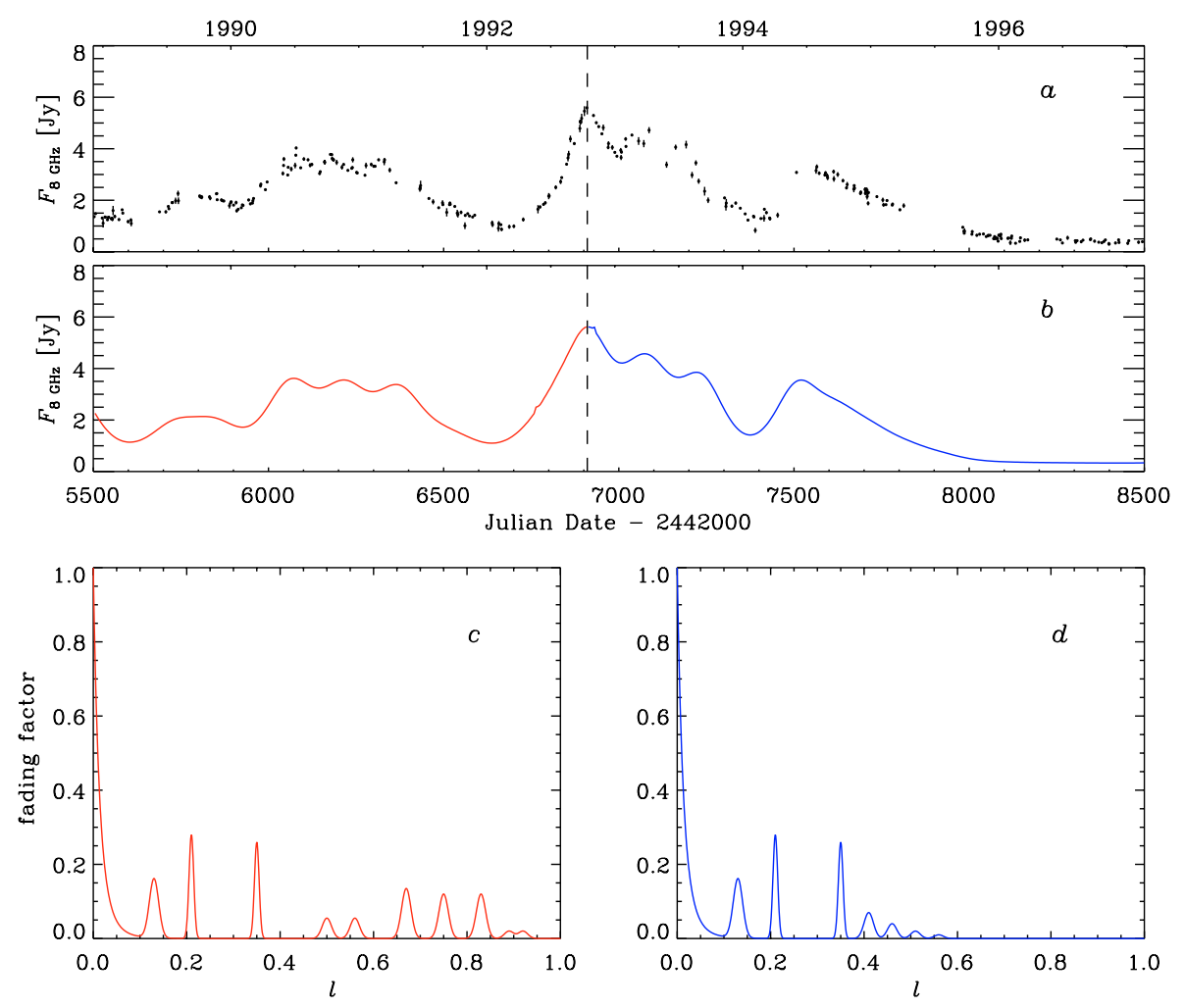

Fig. 8. a) Observed $8 \mathrm{GHz}$ light curve during an 8 -year period centred on the 1992-93 outburst. b) Modelled 8 GHz light curve, under the assumption that non-periodic outbursts originate from enhanced-emission regions. c) Emissivity fading factor before the 1992-93 periodic outburst. d) Emissivity fading factor after the 1992-93 outburst.

are clearly visible in the curve. We can formulate two hypotheses about their origin: (1) they are the effect of some geometric distortions of the helical path; (2) they originate from other phenomena contributing to the source emission.

Looking at the interpolation curve in Fig. 6a, one can see that flux minima are less deep than simulated ones (Fig. 5a), and that they seem to follow a linear decreasing trend down to the lowest state, as previously noticed for the radio light curves of panels b-d, and g of Fig. 1. Hence we defined a "base level" for the source emission, represented by a straight line from the first data point of 1974 (JD $=2442278.6469$ ) to the 1996 deepest minimum (JD = 2450454.7562 ), and by a constant value in the remaining part of the curve (dotted line in Fig. 6a). We then subtracted this trend from the spline, under the assumption that the resulting "cleaned" curve represents the jet contribution alone.

Following hypothesis (1), i.e. supposing the minor outbursts to be due to distortions of the helical path, we derived the time evolution of the local viewing angle $\theta$ corresponding to the cleaned curve. The difference between the result, displayed in Fig. 6b, and the theoretical trend (Fig. 5b) would represent the displacement of the jet path from a perfect helix in the jet region of interest. The presence of the minor outbursts, missing in the modelled light curve, which keeps the mean flux high even when the base level is subtracted, is the reason why the average local viewing angle $\theta$ is low.

Under hypothesis (2), the minor outbursts come from other phenomena in the source. They might be explained as intrinsic variations of the emission, or as due to some more complex geometry of the jet structure implying other contributions to the flux. By removing the non-periodic outburst component from the curve in Fig. 6a, we obtained the shaded light curve of Fig. 7a, and hence the time evolution of $\theta$ shown in Fig. 7b, which looks very similar to that provided by the model, suggesting that the observed periodic behaviour can indeed be accounted for by a rotating jet structure fairly stable in time.

In this latter scenario, we tested the hypothesis that the non-periodic features of the light curve originate from some MHD instabilities occurring in the jet (e.g. pinching modes, which lead to the formation of jet regions characterized by higher-density magnetic field and stronger emissivity), or from other phenomena implying enhanced-emission regions (e.g. shock or magnetic reconnection events).

As the helix rotation goes on, these stronger emitting regions approach the line of sight, giving rise to outbursts in the observed light curve. The presence of enhanced-emission zones along the jet was simulated by modifying the emissivity fading factor represented in Fig. 2 (dashed, red line): Gaussian profiles were added to the curve to reproduce, as an example, the main light curve events observed at $8 \mathrm{GHz}$ over an 8 -year time interval centred on the 1992-93 periodic outburst. The sequence of zones was supposed to be partially evolving during the selected period: the fading factor follows the trend displayed in Fig. 8c before the outburst, and that in Fig. 8d after the outburst. The corresponding modelled light curve, shown in Fig. 8b, well reproduces the observed source behaviour, displayed in Fig. 8a. 


\section{Discussion and conclusions}

We found that the helical-jet model is able to describe the long-term behaviour of the multiwavelength emission of the BL Lacertae object AO 0235+16. Both the periodic occurrence and the mean shape of the main radio and optical outbursts, as well as the corresponding remarkable variations of the broadband SED, can be explained in terms of the orientation change of an inhomogeneous, steadily-emitting, rotating helical jet. The radiation from the different-frequency emitting regions of the jet is affected by relativistic beaming, whose amount depends on the angle between the velocity vector of the emitting plasma and the line of sight, which changes along the helical path and also varies with time. The assumption of flow instabilities (or other phenomena implying local emission enhancement) in the jet provides a viable interpretation for the nonperiodic outbursts observed in the radio light curves.

The twisting of the jet in a helical structure and its rotation can originate from the orbital motion of the parent black hole in a BBHS, the main signature of which would indeed be the periodicity of the source light curves.

The periodicity of AO $0235+16$ is about 2.9 years in the host galaxy rest reference frame, taking into account the $P=$ $P_{\text {obs }} /(1+z)$ relation with $z=0.94$. According to Begelman et al. (1980), this period (assumed to be the orbital period) makes it possible to estimate the mass of the primary black hole for any given value of the mass ratio $M / m$ between the primary and secondary components: $M \sim P_{\mathrm{yr}}^{8 / 5}(M / m)^{3 / 5} 10^{6} M_{\odot}$. For $M / m \sim 1-100$ one can infer $M \sim 5 \times 10^{6}-9 \times 10^{7} M_{\odot}$; the binary separation would be in the range $2 \times 10^{-3}-5 \times 10^{-3} \mathrm{pc}$. If the jet is emitted by the primary, its orbital radius could vary from $5 \times 10^{-5}$ up to $10^{-3} \mathrm{pc}$.

Several VLBI images of the source were produced over a wide range of observing radio wavelengths in the past years. A large fraction of these maps shows no evidence of extended structures apart from the compact core, regardless of the different resolution (Gabuzda et al. 1992; Gabuzda \& Cawthorne 1996); on the other hand, some of them reveal a faint jet north of the core (Jones et al. 1984; Chu et al. 1996; Shen et al. 1997). The presence of a weak extension is confirmed by the sub-milliarcsecond maps obtained with the VSOP at $5 \mathrm{GHz}$ (Frey et al. 2000) and, more recently, with the VLBI at $43 \mathrm{GHz}$ (Jorstad et al. 2001), the latter also displaying a couple of components superluminally moving along bent trajectories.

Our model interprets the jet radio knots as the jet regions where the helical pattern presents the minimum viewing angle, with a maximization of the beaming effect. According to the above orbital radius estimate and taking the pitch angle $\zeta$ and the viewing angle of the helix axis $\psi$ into account, the observed separation between radio knots should be less than $10 \mu$ as, well below the resolution of the most detailed available maps. All this under the assumption that the helix pitch does not vary when moving from the "one-turn" emitting region we considered, which may not be true: the helix pitch could be smaller close to the black hole and increasing outside, up to the observed sub-mas scales.

A huge observing effort is currently going on for this source, in order to closely follow its variability behaviour around the time of the next predicted outburst (first half of 2004; Raiteri et al. 2001): optical and radio telescopes of the WEBT collaboration (http://www.to.astro.it/blazars/webt/; e.g. Villata et al. 2000, 2002, 2004) have been monitoring it since summer 2003, together with the Effelsberg $100 \mathrm{~m}$ radio telescope and VLBA. Moreover, the ground-based observing effort will be intensified in 2004, during the optical/UV/X-ray pointings of the source by the instruments onboard XMM-Newton, which will provide more detail on the shape of the SED in the UV-X-ray band, possibly shedding light on the origin of X-rays in $\mathrm{AO} 0235+16$.

Acknowledgements. We wish to thank the anonymous referee for his/her useful comments, which have helped to clarify a few points of the paper, A. Ferrari for stimulating conversations, R. C. Hartman for useful suggestions on EGRET spectra, E. Trussoni for helpful information about the use of the XSPEC procedure, and A. Comastri for kindly providing us with details on the spectral analysis of the 1993 ROSAT data.

This research has made use of:

- the NASA/IPAC Extragalactic Database (NED), which is operated by the Jet Propulsion Laboratory, California Institute of Technology, under contract with the National Aeronautics and Space Administration;

- the TARTARUS database, which is supported by Jane Turner and Kirpal Nandra under NASA grants NAG5-7385 and NAG5-7067;

- data from the University of Michigan Radio Astronomy Observatory, which is supported by the National Science Foundation and by funds from the University of Michigan.

This work was partly supported by the Italian Ministry for University and Research (MURST) under grant Cofin 2001/028773, by the Italian Space Agency (ASI) under contract CNR-ASI 1/R/27/02, and by the European Community's Human Potential Programme under contract HPRN-CT-2003-00321; it was part of the Ph.D. Thesis of L.O. at the University of Torino.

\section{References}

Abraham, Z. 2000, A\&A, 355, 915

Allen, C. W. 1973, in Astrophysical Quantities, 3rd edition, London: University of London (The Athlone Press)

Aller, H. D., Aller, M. F., Latimer, G. E., \& Hodge, P. E. 1985, ApJS, 59,513

Altschuler, D. R. 1980, AJ, 85, 1559

Altschuler, D. R. 1982, AJ, 87, 387

Altschuler, D. R. 1983, AJ, 88, 16

Altschuler, D. R., \& Wardle, J. F. C. 1976, Mem. R. Astron. Soc., 82, 1

Altschuler, D. R., Broderick, J. J., Condon, J. J., et al. 1984, AJ, 89, 1784

Bååth, L. B., Elgered, G., Lundqvist, G., et al. 1981, A\&A, 96, 316

Begelman, M. C., Blandford, R. D., \& Rees, M. J. 1980, Nature, 287, 307

Bessel, M. S. 1979, PASP, 91, 589

Bloom, S. D., Marscher, A. P., Gear, W. K., et al. 1994, AJ, 108, 398

Brindle, C., Hough, H., Bailey, J. A., Axon, D. J., \& Hyland, A. R. 1986, MNRAS, 221, 739

Briggs, F. H. 1983, AJ, 88, 239

Britzen, S., Roland, J., Laskar, J., et al. 2001, A\&A, 374, 784 
Brown, L. M. J., Robson, E. I., Gear, W. K., et al. 1989, ApJ, 340, 129 Camenzind, M., \& Krockenberger, M. A. 1992, A\&A, 255, 59 Cardelli, J. A., Clayton, C., \& Mathis, J. S. 1989, ApJ, 345, 245 Chu, H. S., Bååth, L. B., Rantakyrö, F. T., Zhang, F. J., \& Nicholson, G. 1996, A\&A, 307, 15

Comastri, A., Fossati, G., Ghisellini, G., \& Molendi, S. 1997, ApJ, 480,534

Condon, J. J., \& Jauncey, D. L. 1974, AJ, 79, 1220

Condon, J. J., Ledden, J. E., O’Dell, S. L., \& Dennison, B. 1979, AJ, 84,1

Cotton, W. D., Geldzahler, B. J., Marcaide, J. M., et al. 1984, ApJ, 286, 503

Cotton, W. D., Fanti, C., Fanti, R., et al. 1997, A\&A, 325, 479

Conway, J. E., \& Wrobel, J. M. 1995, ApJ, 439, 98

Cruz-Gonzales, I., \& Hucra, J. P. 1984, AJ, 89, 441

Edelson, R. A. 1987, AJ, 94, 1150

Edelson, R., Pike, G. F., \& Saken, J. M. 1992, ApJS, 83, 1

Ennis, D. J., Neugebauer, G., \& Werner, M. 1982, ApJ, 262, 460

Epstein, E. E., Landau, R., \& Rather, J. D. G. 1980, AJ, 85, 1427

Frey, S., Gurvits, L. I., Altschuler, D. R., et al. 2000, PASJ, 52, 975

Fugmann, W., \& Meisenheimer, K. 1988, A\&AS, 76, 145

Gabuzda, D. C., \& Cawthorne, T. V. 1996, MNRAS, 283, 759

Gabuzda, D. C., Cawthorne, T. V., Roberts, D. H., \& Wardle, J. F. C. 1992, ApJ, 388, 40

Gear, W. K. 1993, MNRAS, 264, 919

Gear, W. K., Robson, E. I., Ade, P. A. R., et al. 1984, ApJ, 280, 102

Gear, W. K., Robson, E. I., Ade, P. A. R., et al. 1985, ApJ, 291, 511

Gear, W. K., Brown, L. M. J., Robson, E. I., et al. 1986, ApJ, 304, 295

Gear, W. K., Stevens, J. A., Huges, D. H., et al. 1994, MNRAS, 267, 167

Ghisellini, G., \& Maraschi, L. 1989, ApJ, 340, 181

Ghisellini, G., Maraschi, L., \& Treves, A. 1985, A\&A, 146, 204

Ghosh, T., \& Rao, A. P. 1992, A\&A, 264, 203

Ghosh, K. K., \& Soundararajaperumal, S. 1995, ApJS, 100, 37

Ghosh, T., Gopal-Krishna, \& Rao, A. P. 1994, A\&AS, 106, 29

Gregory, P. C., \& Condon, J. J. 1991, ApJS, 75, 1011

Haddock, T. F., \& Aller, M. F. 1987, AJ, 93, 1356

Hartman, R. C., Bertsch, D. L., Bloom, S. D., et al. 1999, ApJS, 123, 79

Holmes, P. A., Brand, P. W. J. L., Impey, C. D., \& Williams, P. M. 1984, MNRAS, 210, 961

Hughes, S. A., \& Blandford, R. D. 2003, ApJ, 505, L101

Impey, C. D., \& Neugebauer, G. 1988, AJ, 95, 307

Impey, C. D., \& Tapia, S. 1990, ApJ, 354, 124

Impey, C. D., Brand, P. W. J. L., Wolstencroft, R. D., \& Williams, P. M. 1982, MNRAS, 200, 19

Impey, C. D., Brand, P. W. J. L., Wolstencroft, R. D., \& Williams, P. M. 1984, MNRAS 209, 245

Jones, T. W., Rudnick, L., Owen, F. N., et al. 1981, ApJ, 243, 97

Jones, D. L., Bååth, L. B., Davis, M. M., \& Unwin, S. C. 1984, ApJ, 284, 60

Jones, D. L., Unwin, S. C., Readhead, A. C. S., et al. 1986, ApJ, 305, 684

Jorstad, S. G., Marscher, A. P., Mattox, J. R., et al. 2001, ApJS, 134, 181

Kaastra, J. S., \& Roos, N. 1992, A\&A, 254, 96

Krichbaum, T. P., Witzel, A., Graham, D. A., et al. 1993, A\&A, 275, 375

Landau, R., Epstein, E. E., \& Rather, J. D. G. 1980, AJ, 85, 363

Landau, R., Jones, T. W., Epstein, E. E., et al. 1983, ApJ, 268, 68

Lawrence, C. R., Readhead, A. C. S., Linfield, R. P., et al. 1985, ApJ, 296, 458

Lichtfield, S. J., Robson, E. I., \& Stebens, J. A. 1994, MNRAS, 270, 341
Madejski, G. M., Takahashi, T., Tashiro, M., et al. 1996, ApJ, 459, 156 Maraschi, L., Ghisellini, G., \& Celotti, A. 1992, ApJ, 397, L5

Mead, A. R. G., Ballard, A. R., Brand, P. W. J. L., et al. 1990, A\&AS, 83,183

Moellenbrock, G. A., Fujisawa, K., Preston, R. A., et al. 1996, AJ, 111,2174

Moles, M., Garcia-Pelayo, J., Masegosa, J., \& Aparicio, A. 1985, ApJS, 58, 255

Mukherjee, R., Bertsch, D. L., Bloom, S. D., et al. 1997, ApJ, 490, 116

Nartallo, R., Gear, W. K., Murray, A. G., Robson, E. I., \& Hough, J. H. 1998, MNRAS, 297, 667

O’Dell, S. L., Puschell, J. J., \& Stein, W. A. 1977, ApJ, 213, 351

O’Dell, S. L., Puschell, J. J., Stein, W. A., \& Warnier, J. W. 1978a, ApJS, 38, 267

O’Dell, S. L., Puschell, J. J., Stein, W. A., et al. 1978b, ApJ, 224, 22

Ostorero, L., Raiteri, C. M., Villata, M., et al. 2001, Mem. Soc. Astron. Ital., 72, 147

Ostorero, L., Villata, M., \& Raiteri, C. M. 2003a, PASPC, 299, 125

Ostorero, L., Villata, M., \& Raiteri, C. M. 2003b, in Proc. Second ENIGMA Meeting, ed. C. M. Raiteri, \& M. Villata, Osservatorio Astronomico di Torino, Pino Torinese, 232

Owen, F. N., Porcas, R. W., Mufson, S. L., \& Moffett, T. J. 1978, AJ, 83,685

Owen, F. N., Spangler, R., \& Cotton, W. D. 1980, AJ, 85, 351

Perley, R. A. 1982, AJ, 87, 859

Press, W. H., Teukolsky, S. A., Vetterling, W. T., \& Flannery, B. P. 1992, Numerical Recipes in Fortran - The Art of Scientific Computing (Cambridge: Cambridge University Press)

Punsly, B. 1997, ApJ, 474, 612

Quirrenbach, A., Witzel, A., Krichbaum, T. P., et al. 1992, A\&A, 258, 279

Raiteri, C. M., Villata, M., \& Tosti, G. 1999, A\&A, 352, 19

Raiteri, C. M., Villata, M., Aller, H. D., et al. 2001, A\&A, 377, 396

Raiteri, C. M., Villata, M., Tosti, G., et al. 2003, A\&A, 402, 151

Raiteri, C. M., et al. 2004, A\&A, in preparation

Reuter, H. P., Kramer, C., Sievers, A., et al. 1997, A\&AS, 122, 271

Rieke, G. H., Grasdalen, G. L., Kinman, T. D., et al. 1976, Nature, 260, 754

Rieke, G. H., \& Lebofsky, M. J. 1985, ApJ, 288, 618

Rieke, G. H., Lebofsky, M. J., Kemp, J. C., Coyne, G. V., \& Tapia, S. 1977, ApJ, 218, L37

Rudnick, L., Jones, T. W., Aller, H. D., et al. 1985, ApJS, 57, 693

Ryle, M., O'Dell, D. M., \& Waggett, P. C. 1975, MNRAS, 173, 9

Salgado, J. F., Altschuler, D. R., Ghosh, T., et al. 1999, ApJS, 120, 77

Salonen, E., Teräsranta, H., Urpo, S., et al. 1987, A\&AS, 70, 409

Schönfelder, V., Bennett, K., Blom, J. J., et al. 2000, A\&AS, 143, 145

Seielstad, G. A., Pearson, T. J., \& Readhead, A. C. S. 1983, PASP, 95, 842

Shen, Z. Q., Wan, T. S., Moran, J. M., et al. 1997, AJ, 114, 1999

Sillanpää, A., Mikkola, S., \& Valtaoja, L. 1991, A\&AS, 88, 225

Simonetti, J. H., \& Cordes, J. M. 1990, ApJ, 349, 97

Sitko, M. L., \& Sitko, A. K. 1991, PASP, 103, 160

Sitko, M. L., Stein, W. A., Zhang, Y. X., \& Wisniewsky, W. Z. 1983, PASP, 95, 724

Sitko, M. L., Schmidt, G. D., \& Stein, W. A. 1985, ApJS, 59, 323

Smith, P. S., Balonek, T. J., Elston, R., \& Heckert, P. A. 1987, ApJS, 64, 459

Sobrito, G., Raiteri, C. M., Villata, M., et al. 2001, in Proc. IV National Meeting on Active Galactic Nuclei, ed. A. Celotti, AGN in the year 2000, Mem. Soc. Astron. Ital., 72, 149

Steppe, H., Salter, C. J., Chini, R., et al. 1988, A\&AS, 75, 317

Steppe, H., Liechti, S., Mauersberger, R., et al. 1992, A\&AS, 96, 441 
Steppe, H., Paubert, G., Sievers, A., et al. 1993, A\&AS, 102, 611 Takalo, L. O., Kidger, M. R., De Diego, J. A., Sillanpää, A., \& Nilsson, K. 1992, AJ, 104, 40

Takalo, L. O., Sillanpää, A., Valtaoja, E., et al. 1998, A\&AS, 129, 577

Teräsranta, H., Tornikoski, M., Valtaoja, E., et al. 1992, A\&AS, 94, 121

Teräsranta, H., Tornikoski, M., Mujunen, A., et al. 1998, A\&AS, 132, 305

Tornikoski, M., Valtaoja, E., Teräsranta, H., et al. 1996, A\&AS, 116, 157

Ulvestad, J. S., \& Johnston, K. J. 1984, AJ, 89, 189

Ulvestad, J. S., Johnston, K. J., \& Perley, R. 1981, AJ, 86, 1010

Ulvestad, J. S., Johnston, K. J., \& Weiler, K. W. 1983, ApJ, 266, 18

Urry, C. M., \& Padovani, P. 1995, PASP, 107, 803

Vetukhnovskaya, Y. N. 1989, AZh, 66, 480

Villata, M., \& Raiteri, C. M. 1999, A\&A, 347, 30
Villata, M., Raiteri, C. M., Sillanpää, A., \& Takalo, L. O. 1998, MNRAS, 293, L13

Villata, M., Mattox, J. R., Massaro, R., et al. 2000, A\&A, 363, 108

Villata, M., Raiteri, C. M., Kurtanidze, O. M., et al. 2002, A\&A, 390, 407

Villata, M., Raiteri, C. M., Kurtanidze, O. M., et al. 2004, A\&A, submitted

Wamsteker, W. 1981, A\&A, 97, 329

Wardle, J. F. K., \& Roberts, D. H. 1988, IAUS, 129, 143

Webb, J. R., Smith, A. G., Leacock, R. J., et al. 1988, AJ, 95, 374

Webb, J. R., Howard, E., Benítez, E., et al. 2000, AJ, 120, 41

Weiler, K. W., \& Johnston, K. J. 1980, MNRAS, 190, 269

Wilson, A. S., \& Colbert, E. J. M. 1995, ApJ, 438, 62

Witzel, A., Schmidt, J., Pauliny-Toth, I. I., \& Nauber, U. 1979, AJ, 84, 942

Worrall, D. M., \& Wilkes, B. J. 1990, ApJ, 360, 396

Xie, G. Z., Li, K. H., Liu, F. K., et al. 1992, ApJS, 80, 683 